Sodium valproate prescribing safety in women of childbearing potential

Shane Donnelly^ and Rajesh Alex

Bassetlaw Mental Health Services

${ }^{*}$ Corresponding author.

doi: 10.1192/bjo.2021.243

Aims. The purpose of this audit was to identify all women being prescribed Sodium Valproate under the Bassetlaw Local Mental Health Team (LMHT) caseload to see how well latest prescribing guidelines are being met, and help set up a system allowing efficient monitoring of Sodium Valproate prescribing in the future. Background. Despite early concerns regarding potential teratogenicity, Sodium Valproate became a widely used anticonvulsant and mood stabiliser and is licensed for use in Epilepsy, Migraine prophylaxis and Bipolar affective disorder. Research evidence now shows its use in pregnancy increases risk of neurodevelopmental disorders to $40 \%$, and serious birth defects to $10 \%$. Despite research finding these risks prescribing practice did not significantly change. To better reflect these findings in clinical practice in 2018 the Pharmacovigilance Risk Assessment Committee recommended Sodium Valproate should not be used in pregnancy unless they have a form of epilepsy unresponsive to other anti-epileptic drugs, and all with childbearing potential should be enrolled in a pregnancy prevention programme (PPP). This was endorsed by UK Medicines and Healthcare Devices Regulatory Agency in April 2018 with launch of the PPP.

Standards:

Must be offered counselling about risks of valproate to unborn child and importance of effective contraception.

Annual specialist Review by a specialist now mandatory

Risk acknowledgement form must be updated at least annually. Method. The electronic RiO records for all female patients on the Bassetlaw LMHT caseload in the year 2019 were checked to identify those prescribed Valproate. For those prescribed Valproate, evidence of annual risk acknowledgement form, date of last appointment, underlying diagnosis and contraceptive method was checked. This data was stored together on an excel file and used to create a patient list to help allow future monitoring.

Result. From 594 female patients identified, 27 (4.5\%) were prescribed Sodium Valproate. Of these, 14 (52\%) had PPP documentation uploaded, 24 (89\%) had been reviewed within the last 12 months, and 13 (48\%) had no documentation of contraceptive method.

Conclusion. This audit helped highlight there is likely a large population of patients not yet on the Pregnancy Prevention Programme. Creating a monitoring system in excel for female patients being prescribed Valproate can help improve adherence to latest guidelines, with a colour coding system to highlight those needing risk acknowledgement forms/appointments within the next three or six months. Educating patients and other healthcare professionals about risks will also help improve prescribing practice and avoid use in pregnancy.

\section{VTE prophylaxis admission assessment full cycle audit and QI project}

Krystyna Drewniak* and Mark Fielding

Dane Garth, Dova Unit

${ }^{*}$ Corresponding author.

doi: 10.1192/bjo.2021.244

Aims. The aim of the project was to assess completion rates for the VTE prophylaxis assessment for patients admitted to Dova Unit,
Dane Garth. Another aim of the project was to identify areas for improvement and changes which could increase compliance rates. Method. In the first cycle of the audit 20 randomly selected patients admitted to Dova Unit, Dane Garth between June and December 2020 were identified and included in the project. Data were then collected from the online patient record system Rio and analysed using an excel spreadsheet.

In the second cycle of the audit 10 randomly selected patients admitted to Dova Unit, Dane Garth between January and February 2021 were identified and included in the project. Data were then collected from the online patient record system Rio, analysed using an excel spreadsheet and compared with the results obtained in the first cycle of the project.

Result. In the first cycle of the audit the overall compliance was found to be $35 \%$. VTE Risk assessment was completed for 50\% of patients included in the study. 'Active VTE on admission' section of the VTE prophylaxis admission assessment was completed for $30 \%$ of patients included in the study. 'Active VTE at 72 hours' section was completed for $20 \%$ of the patients in the study and the 'risk assessment for VTE' form was completed for $40 \%$ of patients included in the study.

In the second cycle of the audit the overall compliance was found to be $50 \%$. VTE Risk assessment was completed for $60 \%$ of patients included in the study. 'Active VTE on admission' section of the VTE prophylaxis admission assessment was completed for $40 \%$ of patients included in the study. 'Active VTE at 72 hours' was completed for $40 \%$ of the patients included in the study and 'risk assessment for VTE' form was completed for $60 \%$ of patients included in the study.

Conclusion. There was an overall improvement in the completion rates for the VTE prophylaxis admission assessment as a result of conducting the project. Working with the junior doctors and other healthcare professionals responsible for completing the VTE prophylaxis admission assessment, we aim to improve our completion rates of vital information even further.

An audit of Individual Care Plan (ICP) in Dublin North City and County (DNCC) child and adolescent mental health service (CAMHS)

Uchechukwu Egbuta*, Cillian Howley, Anitha Selvarajoo, Muhammad Iqbal and Diana Meskauskait

Health Service Executive, Ireland

${ }^{\star}$ Corresponding author.

doi: 10.1192/bjo.2021.245

Aims. The objectives/aims of the Audit include:

1. To standardize and implement ICP for service users attending DNCC CAMHS team in accordance with the established policy.

2. To achieve greater involvement of service users/parents in ICP.

3. To standardize and improve treatment of care involving all members of one team.

Background. Every patient should have a care plan. Each care plan has a set of needs and goals. These are agreed between the service user and key worker and are assessed and measured frequently. Consultation with each service user/parents, as far as practicable is important. Specification of treatment and care required in accordance with best practice should be recorded. Identification of the necessary resources should be recorded and discussed with service user and key worker. Records kept in one 